\title{
Type-I But Not Type-II Interferon Receptor Knockout Mice Are Susceptible to Biliary Atresia
}

\author{
JOACHIM F. KUEBLER, GERARD CZECH-SCHMIDT, JOHANNES LEONHARDT, BENNO M. URE, AND CLAUS PETERSEN
}

Department of Pediatric Surgery, Medical School Hannover, 30625 Hannover, Germany

\begin{abstract}
The etiology of biliary atresia (BA) is not yet understood, but recent studies have shown inflammation with an upregulated interferon (IFN) activity in the intra- and extrahepatic bile ducts of patients with BA. These findings support an inflammatory/ infectious cause of BA as mimicked in our infective murine model. To study the role of the IFN receptors in our model, we used mice with inactivated INF-alpha/beta receptor A129, with inactivated IFNgamma receptor G129, or inactivation of both interferon receptors AG129 as well as the wild type controls W129. Mice were infected with rotavirus within $48 \mathrm{~h}$ of birth and $7 \mathrm{~d}$ postpartum. The incidence of BA in each group was determined during a 3 wk period. In the second week the virus load was measured. BA incidence was $76 \%$ in A129 and 67\% in AG129 animals, whereas in the G129 group only $33 \%$ of the pups developed BA. The wild type presented with a BA-incidence of $15 \%$, while $7 \mathrm{~d}$ old mice failed to develop BA. There was no significant difference in the virus load of the livers between the groups independent of clinical symptoms. In conclusion, inactivation of type I INF-receptor significantly increases the incidence of BA following postpartal rotavirus infection. This effect is independent of the presence of type II-INF-receptors. Thus, in our model a type I IFN-linked deregulation of the innate immune system appears to be crucial for the induction of biliary atresia. (Pediatr Res 59: 790-794, 2006)
\end{abstract}

$\mathrm{B}^{\mathrm{i}}$ liary atresia (BA), a rare cause of the common neonatal jaundice is the most frequent indication for liver transplantation in childhood (1-3). BA is characterized by an ongoing inflammatory process of the intra- and extra hepatic bile ducts combined with a short- or long-distance fibrotic obstruction of the common bile duct. Although symptomatic surgical treatment, such as portoenterostomy (Kasai procedure) or ultimately liver transplantation exists, there is no therapy that can prevent the progression of the disease itself. Therefore, to find and develop curative therapies, it is necessary to investigate and understand the yet unknown etiology of BA.

There are two leading hypotheses of the etiology of BA. The first attributes the development of BA to a malformation of the ductal plate, whereas the second considers BA the consequence of an immune mediated process that occurs perinataly and is triggered by a not yet defined stimulus (4). In

Received June 24, 2005; accepted January 26, 2006.

Correspondence: Claus Petersen, M.D., Department of Pediatric Surgery, Medical School Hannover, Carl-Neuberg-Str. 1, 30625 Hannover, Germany; e-mail: petersen. claus@mh-hannover.de

Supported by Deutsche Forschungsgemeinschaft, DFG.

DOI: $10.1203 / 01 . p d r .0000219860 .96732 .09$ recent years the later theory has gained support as studies have shown evidence of a $\mathrm{T}$ cell mediated inflammation in the liver tissue of diseased patients $(1,5)$.

Balb-c mice develop an atresia of the extrahepatic bile duct if infected postpartally with rotavirus. In this model features similar to those observed in patients with a $\mathrm{T}$ cell mediated inflammation of the intra- and extrahepatic bile ducts were observed $(6,7)$. Shivakumar and coworkers also showed a $\mathrm{T}$ cell related immune response in this model of viral infection and demonstrated that interferon gamma is important in the progression from cholestasis to BA (8). This experimental data corresponds to the up-regulation of interferon gamma in the portal ducts of patients with BA (9). The crucial role of interferon is further supported by our previous finding that timely treatment of infected mice with interferon alpha significantly decreases the incidence of BA (10). Therefore, the effects of interferon alpha and gamma appear to be contrary in our model. Interferon exerts its actions via two receptors the type I interferon receptor consisting of several subtypes such as the interferon $\alpha$ receptor and type II interferon receptor typically activated by interferon $\gamma$. Although clinical and experimental data point to a crucial role of the interferon system, no data exist regarding the function of the interferon receptor for the development of $\mathrm{BA}$. Thus, the aim of this study was to investigate the effects of the selective depletion of interferon receptor type I, type II or both receptors in our model of BA.

\section{MATERIALS AND METHODS}

Animals. All mice were kindly provided by R. Zinkernagel, Institute of Experimental Immunology, Zürich, Switzerland. The mice were kept virusfree in laminar-flow cages, while food, water and litter were sterilized. We used four different mice strains: Mice lacking the type I IFN- $\alpha / \beta$ receptor (A 129), mice lacking type II IFN- $\gamma$ receptor (G 129) or both types of IFN receptors (AG 129) as well as wild types (WT 129). Details about generating these specific strains of mutant mice (deriving from 129/Sv/Ev and C57BL/6) are described elsewhere (11-13). All procedures were in compliance with the national regulations for protection of animals and performed under supervision of the responsible veterinarian in our institution.

Virus. Rhesus Rotavirus of the Serogroup 3 (MMU 18006) was kindly provided by M. Riepenhoff-Talty (14). MA-104 cells were used (embryonic African green monkey, BioWhittaker Biologic Associates, Walkersville, MD) for the cultivation and titration of the virus as previously described $(6,14)$.

Abbreviations: BA, biliary atresia; BD, bile duct; pfu, plaque forming units; RRV, rhesus-rotavirus 
Determination of Virus Load. For virus titration, organs were ultrasonically homogenized (Branson) and centrifuged at $4^{\circ} \mathrm{C}$ and $4000 \mathrm{rpm}$ in a 3350 rotor in a Heraeus Minifuge GL. Titration of the virus in graded, logarithmic dilutions was performed in tissue culture microtitre plates with confluent cells. Readings of the titers was carried out after $4 \mathrm{~d}$ of incubation under standard cell culture conditions (Heraeus incubator, EK/5060/ $\mathrm{CO}_{2}, 5 \% \mathrm{CO}_{2}$ ). Titers were expressed as plaque forming units $(\mathrm{pfu} / \mathrm{mL})(10,11)$.

Animal Trials. All experiments of this study were approved by the government, and performed according to the national regulations for protection of animals.

Infection. Newborn mice were infected with $20 \mu \mathrm{L}$ of a suspension containing $10^{6} \mathrm{pfu}$ virus at approximately $24 \mathrm{~h}$ post partum. Initial experiments were performed using an intraperitoneal application of the virus. However, all infected animals died within few days without development of the phenotype of biliary atresia and we therefore switched to a nasal application in our experiments. As the intranasal application of the virus within $24 \mathrm{~h}$ post partum was associated with about $60 \%$ suffocation ( 7 out of 12 animals), we applied the virus between $24 \mathrm{~h}$ to $48 \mathrm{~h}$ after birth. Using this approach the majority of infected pups survived while the clinical course of the animals with BA was similar to previous studies. To exclude animals that died due to technical problems of the virus inoculation, we excluded all animals that died within $48 \mathrm{~h}$ after the infection. The excluded animals did not show any signs of cholestasis.

Monitoring. Animals were twice daily checked for survival and cholestasis, i.e., icterus of the non fur-covered skin and detection of bilirubin in the urine [Bilugen-Test ${ }^{\mathrm{TM}}$, Boehringer Mannheim] was monitored. All pups which were infected with virus presented with the oily fur syndrome regardless of the development of BA. This phenomenon is well-known to appear in mice, treated with artificially high doses of rotavirus $(6,10,14)$.

Dissection and histology. Mice were killed if they either showed signs of severe illness, such as generalized weakness and immobility, to prevent them from being cannibalized by their mothers, or at 3 wk after infection. All animals killed and animals which were not cannibalized after spontaneous dead (total rate of $>50 \%$ ) were dissected to confirm the diagnosis of biliary atresia. Using a dissection microscope, we observed edematous swelling of the whole extrahepatic bile duct with short or long-segment atresia. Additionally, prestenotic dilatation of the bile duct and hydrops of the gallbladder were seen in several cases. The histologic changes (fixation, paraffin embedding, microsection and HE staining) of the common bile duct and the liver parenchyma, such as parenchymal necrosis, intrahepatic cholangitis, early fibrosis and proliferation of intrahepatic bile ducts were identical to those, which have been described and illustrated elsewhere (8). To investigate the virus load, the liver, the brain and the spleen were harvested for virus titration in separate animals killed at different time points following infection as shown in Table 1.

Treatment with recombinant interferon- $\alpha$. As in previous studies (10), we treated infected animals with recombinant IFN- $\alpha$ (r/IFN- $\alpha$ B/D hybrid;CibaGeigy, Basel). In a first group $(n=13)$, A129 mice were treated six hours after infection with a single-dose injection of INF- $\alpha\left(10^{4} \mathrm{IU}\right.$, i.p. $)$. As we have observed that daily repeated intraperitoneal injection is not well tolerated by small pups, we chose to administer the second dose of IFN- $\alpha$ several days later. Therefore, RRV-infected A129 and AG129 mice received IFN- $\alpha$ at six hours and at six days after the infection $\left(10^{4} \mathrm{IU}\right.$, i.p.). Animals from each group, as well as controls from RRV-infected wild types, A129 and AG129 mice were used to determine the virusload (Table 1).

Statistical analysis. Results in each group are presented in percentages. Statistics were calculated using SPSS statistical analysis software. Differences

Table 1. Virusload of liver and brain

\begin{tabular}{llccc}
\hline $\begin{array}{c}\text { Mouse } \\
\text { strain }\end{array}$ & $\begin{array}{c}\text { IFN- } \alpha \\
\text { treatment }\end{array}$ & $\begin{array}{c}\text { Days post } \\
\text { infection }\end{array}$ & Cholestasis/BA & $\begin{array}{c}\text { Virusload (pfU) } \\
\text { Liver/brain }\end{array}$ \\
\hline W129 & no & 12 & yes & $10^{4} / 10^{3}$ \\
W129 & no & 12 & no & $10^{4} / 10^{4}$ \\
A129 & no & 12 & yes & $10^{4} / 10^{4}$ \\
AG129 & no & 12 & yes & $10^{3} / 10^{4}$ \\
AG129 & no & 12 & no & $10^{4} / 10^{3}$ \\
A129 & 6 hours p.i. & 5 & no & $10^{3} / 10^{3}$ \\
A129 & 6 hours +6 days & 9 & yes/no & $10^{3} / 10^{4}$ \\
& p.i. & & & \\
\hline
\end{tabular}

Different IFN-receptor knockout strains and wild type controls were infected on the second day of life via intranasal ingestion of a RRV-virus suspension and cholestasis and survival were monitored. were analyzed by Kaplan-Meier-survival calculated for all groups and Kruskal-Wallis ANOVA on ranks followed by pairwise comparison using Dunn's method. Statistical significance was accepted at $p<0.05$.

\section{RESULTS}

Incidence of biliary atresia in Ifn-receptor knockout mice. We observed in our animals similar features of biliary atresia and a similar course of the disease as has been described in the infected Balb/c-mice (Fig. 1) and (6). In the wild-type group, 16 pups out of $54(30 \%)$ had persisting signs of cholestasis with a lethality of $50 \%$ (Fig. 2). Deceased animals were dissected and a variety of BA-forms (Fig. 3), as described previously (6), was observed, so that biliary atresia was confirmed as cause of death of all cholestatic animals that died within the observation period.

We observed significant differences in the development of biliary atresia between the wild type and the INF-receptorknockout mice. Animals lacking the type I INF-receptor alone or in combination with the type II INF-receptor had a significantly higher incidence $(p<0.05)$ of cholestasis compared with wild type controls (Fig. 2). The depletion of only the type II receptor did not signficantly change the incidence of cholestasis (Fig. 2). Compared with the incidence of BA in the wild type mice $15 \%$ ( 8 out of 54 ), mice lacking the type I INF-receptor A129 developed BA in 76\% (51 out of 67) and mice lacking type I INF-receptor in combination with type II receptor G129 in 67\% (22 out of 33) (Fig. 1). This was a significant increase $(p<0.05)$. The incidence of biliary atresia $(33 \%, 19$ out of 57$)$ in the group deficient only in the type II IFN-receptor G129 was significantly increased in comparison to wild types $(p<0.05)$, but significantly lower than in type I and type I and II interferon receptor knockouts $(p<0.05)$ (Fig. 1).

Effects of interferon- $\alpha$ treatment in IFN-receptor knockout mice. In the first group of experiments, IFN type I receptor knockout mice A129 were treated with a single-dose of 10,000 IU IFN- $\alpha$. In this group eight out of thirteen pups became icteric, and of those mice, six finally died (lethality 46\%) (data not shown).

In a second group, twenty-four A129 pups were treated with a 2-fold injection of 10,000 IU IFN- $\alpha$. The lethality in this

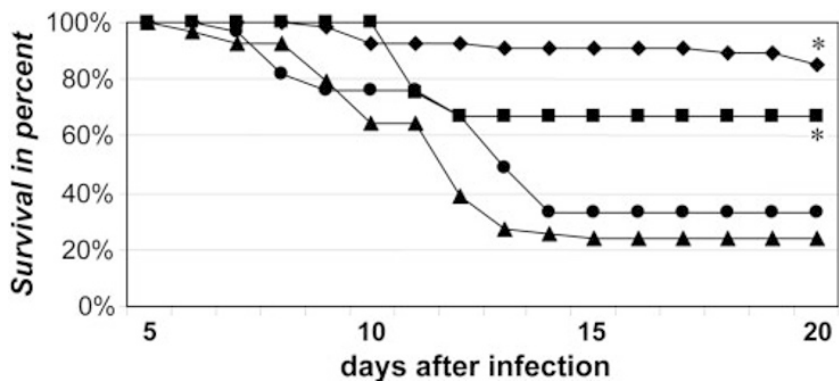

Figure 1. Survival. Survival rate of different interferon receptor knockout mice. Wild types W129 ( ), type I interferon receptor knockouts A129 ( $(\mathbf{\Delta})$,

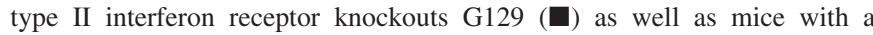
combined type I and II interferon receptor deficiency AG129 (-) were infected with RRV virus and the survival monitored. Statistical analysis of the overall survival rate using Kaplan Meier survival analysis. ${ }^{*} p<0.05$ vs all other groups 


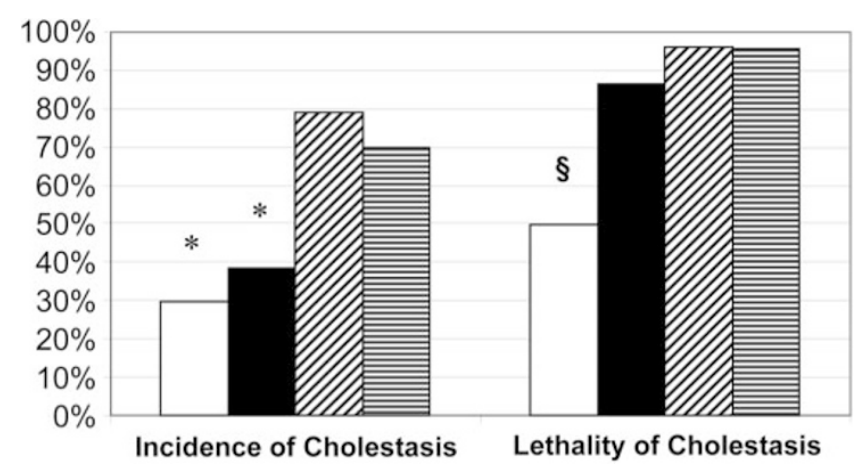

Figure 2. Incidence of cholestasis and lethality of cholestasis. The incidence of cholestasis and the incidence of biliary atresia in cholestatic mice (= lethality of cholestasis) in wild types W129 ( $\square$ ), type I interferon receptor

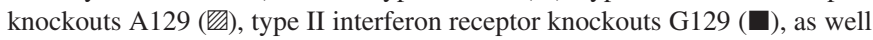
as mice with a combined type I and II interferon receptor deficiency AG129 (冒), infected with RRV virus was daily monitored. * $p<0.05$ vs A129 and AG129. $\$ p<0.05 v s$ all other groups.

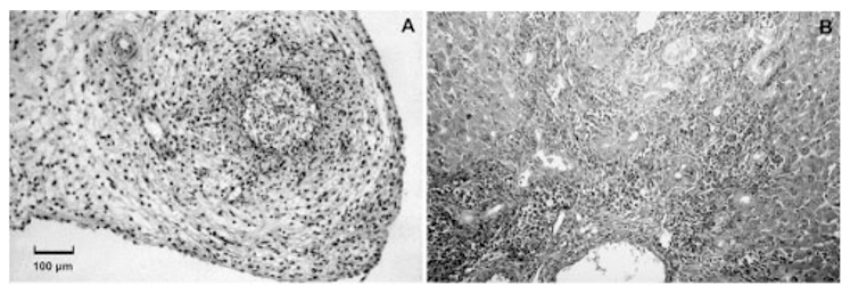

Figure 3. Morphology of biliary atresia. Animals underwent histologic examination post mortem. Cryosections of livers harvested immediately after the deaths of the animals were stained with an HE stain. Original magnification 200x, bar represents $100 \mu \mathrm{m}$. Atresia of the bile ducts $(A)$ and inflammatory reaction $(B)$ were confirmed.

group decreased to $54 \%$ compared with $76 \%$ lethality in to the respective untreated group, and this difference was statistically significant (Fig. 4a). This was associated with a higher recovery rate of cholestatic mice, following the repeated IFNtreatment in the type I IFN-receptor knockout mice (Fig. 4b).

IFN- $\alpha$ treated AG129 mice presented with a lethality of $62 \%$, not different to the $67 \%$ lethality in the untreated AG129 controls, (Fig. 5a). All nonsurvivors were cholestatic and died between day 10 and 21 after infection with the typical clinical and histomorphological signs of BA, as described above. There was no significant difference in the incidence and lethality of cholestasis in the AG129 mice with and without interferon treatment (Fig. 5b).

Determination of virus load. In 12 mice of each wild-type, A129 and AG129 following RRV-infection, the levels of virus in liver and brain tissues were between $10^{3}$ or $10^{4} \mathrm{pfU}$, independent of the clinical symptoms. G129-pups which had received 10,000 IU IFN $6 \mathrm{~h}$ after RRV-infection presented with the identical virus load in liver and brain. At dissection, none of these animals had developed the full picture of biliary atresia. A129-pups which had been treated by repetitive injection of 10,000 IU IFN were also found to have a virus load between $10^{3}$ and $10^{4} \mathrm{pfU}$ RRV in liver and brain. In this group, one animal had the features of biliary atresia [table 1].

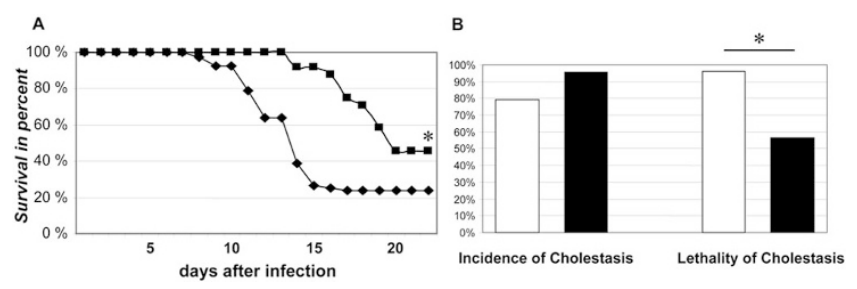

Figure 4. Treatment of type I interferon receptor knockout mice with interferon- $\alpha$. (A) Survival rate of type I interferon receptor knockout mice (A129) treated twice with interferon alpha i.p. $(\square)$ and untreated controls $(\diamond)$. $(B)$ Incidence of cholestasis and incidence of biliary atresia in cholestatic mice (= lethality of cholestasis) in type I interferon receptor knockout mice (A129) treated twice with interferon alpha i.p. (四) and untreated controls ( $\square$ ). Statistical analysis of the overall survival rate using Kaplan Meier survival analysis. $* p<0.05$ vs untreated A129.

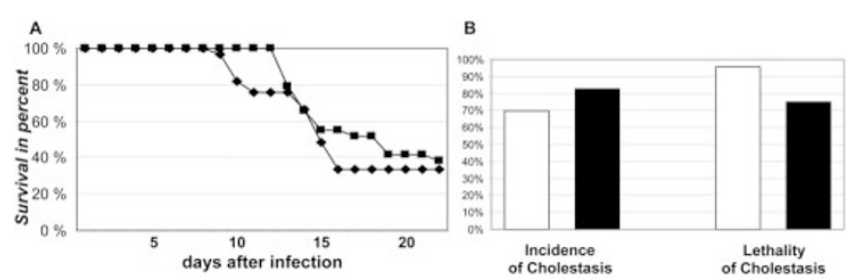

Figure 5. Treatment of type I and II interferon receptor knockout mice with interferon- $\alpha$. (A) Survival rate of type I and II interferon receptor knockout

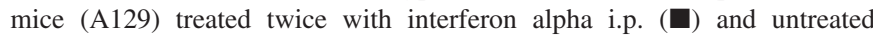
controls $(\checkmark)$. $(B)$ Incidence of cholestasis and incidence of biliary atresia in cholestatic mice (lethality of cholestasis) in type I and II interferon receptor knockout mice (A129) treated twice with interferon alpha i.p. (ם) and untreated controls $(\square)$. Statistical analysis of the overall survival rate using Kaplan Meier survival analysis.

\section{DISCUSSION}

The hypothesis of an inflammatory etiology of BA is based on Landing's theory that the clinical picture of BA represents the final state of an inflammatory process, triggered e.g., by an anticipated viral infection (5). In this regard, investigations have found signs of an inflammatory process in patients with BA, namely $\mathrm{T}$ cell activation, up-regulation of cytokines of innate immunity and intercellular adhesion molecules that support Landing's theory. Nonetheless, several studies have failed to identify a specific hepatotropic virus in patients with $\mathrm{BA}$ and existing studies describing the pathomechanism of $\mathrm{BA}$ are inconclusive $(15,16)$. These findings raised the question of potential autoimmunological co-mechanisms (17) and Perlmutter finally, proposed that BA, rather than being a single disease could represent a common feature initiated by a variety of different etiologic factors (18). To investigate the etiology of BA we developed a mouse model of biliary atresia based on observations of M. Riepenhoff Talty $(6,14,19)$.

In this model, B.A. can be induced by infection with Rhesus Rotavirus only within a short-time period after birth, which suggests an immunologic gap in this period. Among the tested animals, Balb/c-mice are the most susceptible for RRVinduced BA. Balb/c mice do not express Mx-proteins, interferon-induced GTPases with an antiviral activity against a wide range of RNA viruses. An important role of the interferon system in this model is further supported by our observation that repeated IFN- $\alpha$ treatment is curative. We therefore 
investigated the role of the interferon system in our model of BA. We found that newborn animals did not tolerate the repetitive i.p. injection of IFN-antibodies [Petersen C, unpublished observation] and thus, we chose to use type I and type II IFN-receptor knockout mice generated by Zinkernagel and coworkers in the Institute of Experimental Immunology Zürich, Switzerland, to specifically elucidate the roles of type I and type II interferons. The type I IFNs (IFN- $\alpha$, IFN- $\beta$, IFN- $\omega$ ) are encoded by a family of over 20 genes that bind to a common receptor, composed of several subunits (13). One of these receptor components is essential for the functional response to IFN- $\alpha,-\beta$ and $-\omega$. The type II interferon receptor is activated predominantly by IFN- $\gamma$, although there is some crossreactivity between IFNs type I and type II (11-13). Zinkernagel and coworkers observed in the type I IFNreceptor knockout mice A129 an increased susceptibility to different viruses compared with wild type mice and mice with only a nonfunctional type II IFN receptor. Previous studies have demonstrated that both IFN receptors are not necessary to protect or recover from an infection with a pneumotropic influenza virus in mice. However, increased antibody responses were noted following the disruption of IFN type I receptor (20) and it is known that the functional type I IFN-system has an impact on the early antiviral defense and is associated with a reversible depression of hematopoesis (21). Moreover, Salazar-Mather and coworkers have demonstrated that in the livers of adult mice infected with murine cytomegalovirus type I IFNs control the recruit of inflammatory cells (22). The IFN- $\gamma$ system appears to play a more important role in later stages of the response to viral infections (23). Using adult mice Zinkernagel and coworkers observed no significant effect of IFN-treatment on rotavirus induced diarrhoea and viral replication in these IFN-mutant mice (11-13). In this regard, age-related studies in mice, which were infected with rotavirus, revealed an age restriction of clinical symptoms $(24,25)$ and therefore this data cannot be easily transferred to our model of infection of the newborn.

In the present study, newborn mice of the IFN-mutant strains and its wild type were more susceptible to RRV than other inbred mouse strains (19). High lethality occurred in the pups which were intraperitonally infected within their first week of life and none of these mice survived to develop biliary atresia. We know from studies with Balb/c mice, that BA can only be induced if the infection is performed during the first days of life (10) and accordingly pups infected in their second week of life survived with only signs of rotavirus induced diarrhea but no cholestasis. Thus, the approach of the intraperitoneal virus ingestion failed to induce biliary atresia in these mice. After changing the route of infection, mice were able to survive and to develop biliary atresia, most likely because of the different kinetics of the virus distribution. The incidence of cholestasis and biliary atresia $(15 \%)$ in the wild type W129 was lower than in Balb/c-mice (BA-incidence approx. 80\%) and comparable to the reported incidence in CD-mice (BA-incidence approx. 33\%) (19).

All mice defective in type I and/or type II interferon receptor showed a significantly reduced survival rate, i.e., significantly increased incidence of biliary atresia. This sup- ports the previously postulated major role of the interferon system in the development of BA. However, we observed that animals with no functional type I interferon receptor alone or in combination with a nonfunctional type II receptor had a significantly increased mortality. The increased lethality was associated with a significant increase of cholestatic mice in the type I IFN knockouts A129 and in type I and II knockouts AG129, compared with wild types, but not in animals only lacking the type II IFN receptor G129. Interestingly, all knock-out mice showed a significantly increased lethality of cholestasis, i.e., the progression of cholestasis to biliary atresia was significantly higher with the lack of either interferon receptors. Taken together these findings suggest that specifically lack of the type I receptor makes animals susceptible to the development of biliary atresia. As the type I IFN system is well known as a first-line antiviral agent (26), it could be speculated that the increase in lethality is due to the inability of the type I interferon knockouts to defend themselves against the viral infection, rather than a direct involvement in the pathogenesis of BA. However, it should be noted that there was no significant difference in virus load in the livers and brains of IFN-receptor deficient mice with and without biliary atresia. This observation that is in line with previous studies in Balb/c-mice demonstrating that BA-development is triggered by RRV, but is not correlated to the virus load in the liver (10). Therefore it appears that the interferon type I system plays an important role in the development of BA, rather than just improving the defense against the viral infection, i.e., reducing the virus load. Several known properties of type I IFN could contribute to this effect. Besides actions that are redundant to the type II IFN system, such as the promotion of a switch in Ig class with an increased production of virus specific $\operatorname{IgG}$ antibodies, type I interferons exhibit unique and partially antagonistic effects compared with type II interferons. For example type I interferons have been shown to dampen the inflammatory response in the liver potentially via increased levels of IL-10 and sCD14 as well as an increase in CD 14 positive macrophages in the liver.

Deficiency in type II IFN receptor did not affect the incidence of BA as strong as the lack of type I IFN receptor in our model, but still led to a significantly increased incidence of biliary atresia. This was mediated not by an increase in the incidence, but by a significantly reduced recovery rate of cholestatic mice. This suggests that in our model interferon $\gamma$ is involved in the recovery from cholestasis. This appears to be in contrast to studies by Shivakumar et al., who demonstrated that the deficiency of interferon- $\gamma$ is associated with a reduced incidence of biliary atresia and an improved long term survival using this model in balb-c mice (27). Moreover they showed that intraperitoneal administration of interferon- $\gamma$ could increase the incidence of biliary atresia in the knock out mice to levels of the controls, and conclude that interferon- $\gamma$ could play an important role in the transition of cholestasis to biliary atresia $(9,27)$. The cause for this difference in the effects of the type II interferon system remains to be determined. However, there are interactions between the two interferon receptor systems on the signal transduction levels that makes the comparison of the interferon gamma knockout mice 
with type II interferon receptor knockout mice difficult (28) and possible explanations could include reactive adaptions in the knock out mice as well as nonreceptor specific effects of interferon- $\gamma$.

Previous studies have demonstrated that Balb/c-mice treated with interferon-alpha are protected against the virus induced cholestasis and development of biliary atresia (10). In contrast, repetitive treatment with recombinant interferon- $\alpha$ of RRV-infected A129 and AG129 mice did not affect the incidence of cholestasis in type I IFN receptor deficient and type I and II IFN receptor deficient pups, although the survival rate of A129 mice was increased. The diminished protection observed in type I and type I and II receptor knockouts supports the hypothesis that the beneficial effects of interferon- $\alpha$ treatment are mediated by activation of the type I interferon receptor. The increased survival of A129 mice following interferon- $\alpha$ treatment was related to an improved recovery from cholestasis. It could be speculated that the high dose of interferon- $\alpha$ could directly or indirectly activate type II interferon receptors in mice only deficient for the type I interferon receptor. This interpretation would fit the notion that activation of type II interferon receptors supports the recovery from cholestasis and can prevent the transition from cholestasis to biliary atresia.

In conclusion, our results demonstrate an important role of the type I and type II interferon receptors for the prevention of biliary atresia in our model. Type I receptors appear to be crucial in the prevention of cholestasis as well as the recovery. The type II IFN receptor activation although less important than the type I receptors appears to contribute to the recovery of cholestatic pups. Further studies are needed to demonstrate whether beneficial effects of the interferon receptors are mediated via an improved innate immune response, or whether the activation of these receptors is directly part of the pathogenesis of biliary atresia.

Acknowledgments. We thank R. Zinkernagel, Zürich, for providing us with W129, A129, G129, and AG129 mice, P. Staeheli, Freiburg, for critical and innovative, and C. Börner for linguistic reviewing of the manuscript.

\section{REFERENCES}

1. Balistreri WF, Grand R, Hoofnagle JH, Suchy FJ, Ryckman FC, Perlmutter DH, Sokol RJ 1996 Biliary atresia: current concepts and research directions. Summary of a symposium. Hepatology 23:1682-1692

2. Haber BA, Russo P 2003 Biliary atresia. Gastroenterol Clin North Am 32:891-911
3. Sokol RJ, Mack C, Narkewicz MR, Karrer FM 2003 Pathogenesis and outcome of biliary atresia: current concepts. J Pediatr Gastroenterol Nutr 37:4-21

4. Kobayashi H, Stringer MD 2003 Biliary atresia. Semin Neonatol 8:383-391

5. Landing BH 1974 Consideration of the pathogenesis of neonatal hepatitis, biliary atresia and choledochal cyst - the concept of infantile obstructive cholangiopathy. Prog Pediatr Surg 113-139.

6. Petersen C, Biermanns D, Kuske M, Schakel K, Meyer-Junghanel L, Mildenberger H 1997 New aspects in a murine model for extrahepatic biliary atresia. J Pediatr Surg 32:1190-1195

7. Czech-Schmidt G, Verhagen W, Szavay P, Leonhardt J, Petersen C 2001 Immunological gap in the infectious animal model for biliary atresia. J Surg Res 101:62-67

8. Shivakumar P, Campbell KM, Sabla GE, Miethke A, Tiao G, McNeal MM, Ward RL, Bezerra JA 2004 Obstruction of extrahepatic bile ducts by lymphocytes is regulated by IFN-gamma in experimental biliary atresia. J Clin Invest 114:322-329

9. Bezerra JA, Tiao G, Ryckman FC, Alonso M, Sabla GE, Shneider B, Sokol RJ, Aronow BJ 2002 Genetic induction of proinflammatory immunity in children with biliary atresia. Lancet 360:1653-1659

10. Petersen C, Bruns E, Kuske M, von Wussow P 1997 Treatment of extrahepatic biliary atresia with interferon-alpha in a murine infectious model. Pediatr Res 42:623-628

11. Huang S, Hendriks W, Althage A, Hemmi S, Bluethmann H, Kamijo R, Vilcek J, Zinkernagel R, Aguet M 1993 Immune response in mice that lack the interferon- $\gamma$ receptor. Science 259:1742-1745

12. Müller U, Steinhoff U, Reis L, Hemmi S, Pavlovic J, Zinkernagel RM, Aguet M 1994 Functional role of type I and type II interferons in antiviral disease. Science 264:1918-1921

13. van den Broek M, Müller U, Huang S, Aguet M, Zinkernagel RM 1995 Antiviral defense in mice lacking both alpha/beta and gamma interferon receptors. J Virol 69:4792-4796

14. Riepenhoff-Talty M, Schaekel K, Clark HF, Mueller W, Uhnoo I, Rossi T, Fisher J, Ogra PL 1993 Group A rotaviruses produce extrahepatic biliary obstruction in orally inoculated newborn mice. Pediatr Res 33:394-399

15. Urushihara N, Iwagaki H, Yagi T, Kohka H, Kobashi K, Morimoto Y, Yoshino T, Tanimoto T, Kurimoto M, Tanaka N 2000 Elevation of serum interleukin-18 levels and activation of Kupffer cells in biliary atresia. J Pediatr Surg 35:446-449

16. Minnick KE, Kreisberg R, Dillon PW 1998 Soluble ICAM-1 (sICAM-1) in biliary atresia and its relationship to disease activity. J Surg Res 76:53-56

17. A-Kader HH, Abdel-Hameed A, Al-Shabrawi M, Mohsen N, El-Karaksy H, Hassanein B, Elsayed B, Abdel-Khalik MK, Karjoo M 2003 Is biliary atresia an autoimmune disease? Eur J Gastroenterol Hepatol 15:447-

18. Perlmutter DH, Shepherd RW 2002 Extrahepatic biliary atresia: a disease or a phenotype? Hepatology 35:1297-1304

19. Petersen C, Kuske M, Bruns E, Biermanns D, Wussow PV, Mildenberger H 1998 Progress in developing animal models for biliary atresia. Eur J Pediatr Surg 8:137-141

20. Price GE, Gaszewska-Mastarlarz, Moskophidis D 2000 The role of alpha/beta and gamma interferons in development of immunity to influenza A virus in mice. J Virol 74:3996-4003

21. Binder D, Fehr J, Hengartner H, Zinkernagel RM 1997 Virus-induced transient bone marrow aplasia: major role of interferon- $\alpha / \beta$ during infection with the noncytopathic lymphocytic choriomeningitis virus. J Exp Med 185:517-530

22. Salazar-Mather TP, Lewis CA, Biron CA 2002 Type I interferons regulate inflammatory cell trafficking and macrophage inflammatory protein 1alpha delivery to the liver. J Clin Invest 110:321-330

23. Khan S, Zimmermann A, Basler M, Groettrup M, Hengel H 2004 A cytomegalovirus inhibitor of gamma interferon signaling controls immunoproteasome induction. J Virol 78:1831-1842

24. Rose J, Franco M, Greenberg H 1998 The immunology of rotavirus infection in the mouse. Adv Virus Res 51:203-235

25. Vancott JL, McNeal MM, Choi A H, Ward RL 2003 The role of interferon in rotavirus infection and protection. J Interferon Cytokine Res 23:163-170

26. Pestka S, Krause CD, Walter MR 2004 Interferons, interferon-like cytokines, and their receptors. Immunol Rev 202:8-32

27. Shivakumar P, Campbell KM, Sabla GE, Miethke A, Tiao G, McNeal MM, Ward RL, Bezerra JA 2004 Obstruction of extrahepatic bile ducts by lymphocytes is regulated by IFN-gamma in experimental biliary atresia. J Clin Invest 114:322-329

28. Takaoka A, Mitani Y, Suemori H, Sato M, Yokochi T, Noguchi S, Tanaka N, Taniguchi T 2000 Cross talk between interferon-gamma and -alpha/beta signaling components in caveolar membrane domains. Science 288:2357-2360 